\title{
Simultaneous Occurrence of Double-Hit Lymphoma and Acute Myeloid Leukemia in Bone Marrow
}

\author{
Daisuke Sakamoto ${ }^{1}$, Norihide Satoh ${ }^{2}$, Hiroaki Ohnishi ${ }^{1 *}$, Satoko Yamasaki ${ }^{1}$, Takashi Watanabe ${ }^{1}$ and Nobuyuki Takayama ${ }^{2}$ \\ ${ }^{1}$ Department of Laboratory Medicine, Kyorin University, Shinkawa, Mitaka, Tokyo, Japan \\ ${ }^{2}$ Department of Hematological Medicine, Kyorin University, Shinkawa, Mitaka, Tokyo Japan
}

\begin{abstract}
An 81-year-old man presented at hospital with general fatigue, loss of body weight, and high fever. A blood examination showed anemia, thrombocytopenia, neutrophil dysplasia, and the presence of blastoid cells. Bone marrow aspiration also revealed the presence of blast cells and dysmorphic lymphocytes. Flow cytometric analysis of bone marrow cells identified three distinct aberrant cell populations: small and large cells both positive for CD10, CD19 and CD20, and medium-sized to large cells positive for CD13, CD34, and CD117. Cytogenetic analysis of the bone marrow cells showed two distinct populations having either $46, \mathrm{XY}, \mathrm{t}(8 ; 22)(\mathrm{q} 24 ; \mathrm{q} 11.2), \mathrm{t}(14 ; 18)(\mathrm{q} 32 ; \mathrm{q} 21)$ or $46 \mathrm{XY},-5,-17,-20,+$ mar1, +mar2, +mar3. This is an extremely rare case with concurrence of DHL and AML, and his lymphoma cells and leukemia cells in the bone marrow were difficult to distinguish morphologically. It is essential to fully perform cytogenetic analysis and immunophenotyping for accurate evaluation of bone marrow involvement of DHL.
\end{abstract}

\section{Introduction}

Double-hit lymphoma (DHL) is a disease entity recently introduced to describe malignant lymphoma with two recurrent translocations typically involving MYC and BCL2 oncogenes [1-3]. The term DHL is also used to refer to lymphomas having abnormalities of other oncogenes such as BCL6 concurrent with MYC translocation, or those having corresponding translocations demonstrated by cytogenetics. Several studies with a large series of DHL cases have clarified its aggressive disease course and poor prognosis [4-7]. Of note, approximately $40 \%$ of DHL patients have evidence of bone marrow involvement, with $10 \%$ of cases showing leukemic presentation. A large retrospective study revealed that both bone marrow involvement and leukemic presentation are prognostic factors predicting shorter event free survival and overall survival [4]. Therefore, accurate evaluation of bone marrow involvement is essential for better prediction of disease course and establishment of treatment strategy in DHL.

Morphologically, majority of tumor cells of DHL show features intermediate between Burkitt lymphoma and diffuse large B-cell lymphoma (DLBCL), but a subset of cases with features of B-lymphoblastic lymphoma or follicular lymphoma has also been identified [8]. Consequently, lymphoma cells infiltrating in bone marrow can show wide variety of morphology, leading to difficulty in diagnosis of bone marrow involvement. Here we describe a rare case of DHL with bone marrow involvement that showed concurrent development of acute myeloid leukemia (AML). Although morphological distinction between lymphoma cells and leukemic cells in the bone marrow was difficult, cytogenetic analysis and immunophenotyping by flow cytometry (FCM) were useful in discriminating these two types of malignant hematological cells.

\section{Case Report}

An 81-year-old man presented at hospital with general fatigue, loss of body weight, and high fever. He had been suffering from chronic anemia and thrombocytopenia for years, but precise hematological evaluation had not been performed. The blood examination at the hospital showed the presence of blastoid cells in his peripheral blood, and he was referred to our hospital. Physical examination revealed no specific symptoms except for anemia in palpebrae. The laboratory evaluation revealed elevated serum lactate dehydrogenase level of 923 IU/L (reference range: 124-222). Computed tomography disclosed axillary lympadenopathy and abnormal para-aortic mass. A hematological examination showed mild anemia (hemoglobin level of $103 \mathrm{~g} / \mathrm{L}$ ) and thrombocytopenia (platelet count of $95.0 \times 10^{9} / \mathrm{L}$ ), neutrophil dysplasia, and the presence of blastoid cells (21.5\% of white blood cells) and dysmorphic lymphocytes (2.5\% of white blood cells). Bone marrow aspiration revealed the presence of blast cells $(6.6 \%$ of total nucleated cells (TNCs)) and dysmorphic lymphocytes $(76.6 \%$ of TNCs). The blast cells were medium-sized to large with basophilic cytoplasm, and some contained nucleoli on the nucleus (Figure 1).

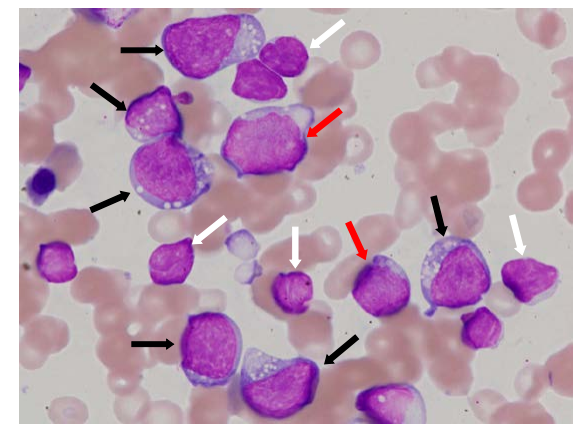

Figure 1: Wright-Giemsa staining of cell smears of bone marrow aspiration $(\times 1000)$.

The blast cells were medium-sized to large with basophilic cytoplasm, and some contained nucleoli on the nucleus (red arrows). Dysmorphic lymphocytes were of two types: small cells with a high nucleus/cytoplasm ratio, some of which had deeply cleaved nuclei (white arrows); and large cells with basophilic cytoplasm, some of which contained a few cytoplasmic vacuoles (black arrows).

*Corresponding author: Ohnishi $\mathrm{H}$, Department of Laboratory Medicine, Kyorin University, Shinkawa, Mitaka, Tokyo 181-8611, Japan, Tel: +81-422-47-5511; Fax: +81-422-79-3471; E-mail: onishi@ks.kyorin-u.ac.jp

Received November 28, 2017; Accepted November 30, 2017; Published December 02, 2017

Citation: Sakamoto D, Satoh N, Ohnishi H, Yamasaki S, Watanabe T, et al. (2017) Simultaneous Occurrence of Double-Hit Lymphoma and Acute Myeloid Leukemia in Bone Marrow. J Blood Lymph 7: 188. doi: 10.4172/2165-7831.1000188

Copyright: (c) 2017 Sakamoto D, et al. This is an open-access article distributed under the terms of the Creative Commons Attribution License, which permits unrestricted use, distribution, and reproduction in any medium, provided the original author and source are credited. 
Citation: Sakamoto D, Satoh N, Ohnishi H, Yamasaki S, Watanabe T, et al. (2017) Simultaneous Occurrence of Double-Hit Lymphoma and Acute Myeloid Leukemia in Bone Marrow. J Blood Lymph 7: 188. doi: 10.4172/2165-7831.1000188

Dysmorphic lymphocytes were of two types: small cells with a high nucleus/cytoplasm ratio, some of which had deeply cleaved nuclei, accounting for $40 \%$ of TNCs; and large cells with basophilic cytoplasm, some of which contained a few cytoplasmic vacuoles, accounting for $36.6 \%$ of TNCs. However, blast cells and large dysmorphic lymphocytes were sometimes difficult to distinguish. Myeloperoxidase was weakly positive in $2 \%$ of bone marrow blastoid cells, while esterase and periodic acid-Schiff (PAS) staining was negative. Histopathological examination of the bone marrow biopsy specimen revealed two types of lymphoid cell populations, with small cells having a round nucleus and large cells with vacuoles, both of which were positive for CD10, CD20, CD79a, bcl-2, and bcl- 6 by immunohistochemistry. Biopsy of the lymph nodes or para-aortic mass was not performed due to deteriorating conditions of the patient.

CD10-, CD19-, CD20-, and HLA-DR-positive populations of bone marrow cells were detected by flow cytometry in the area of CD45positive populations of large and small cells which accounted for $29.5 \%$ and $36.1 \%$ of TNCs, respectively. A distinct population of mediumsized to large cells (7.2\% of TNCs) positive for CD13, CD34, CD117, and HLA-DR was detected in the area of cells dimly positive for CD45 (Figure 2).

A
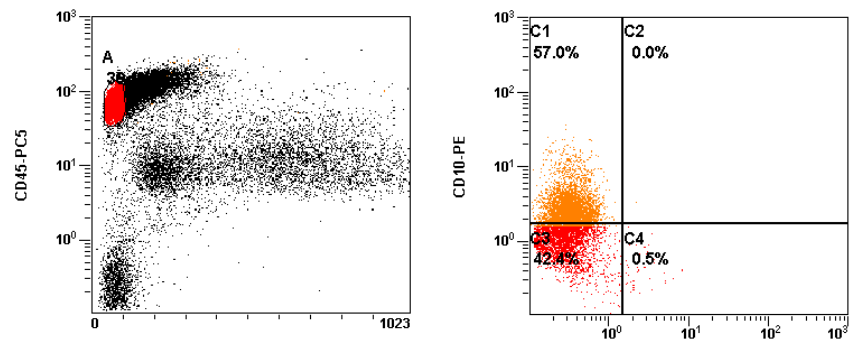

Ss Lin

B

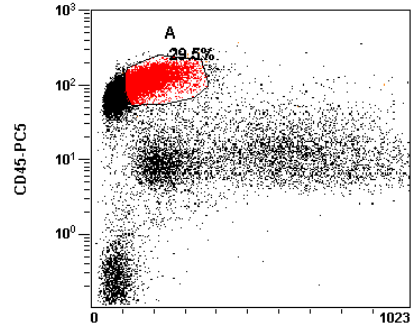

SS Lin

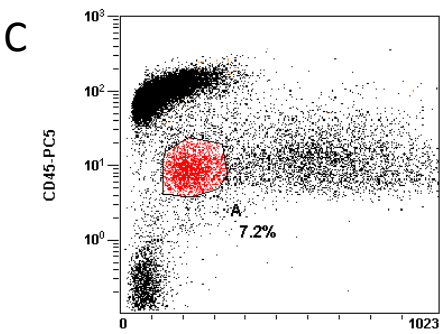

ss Lin
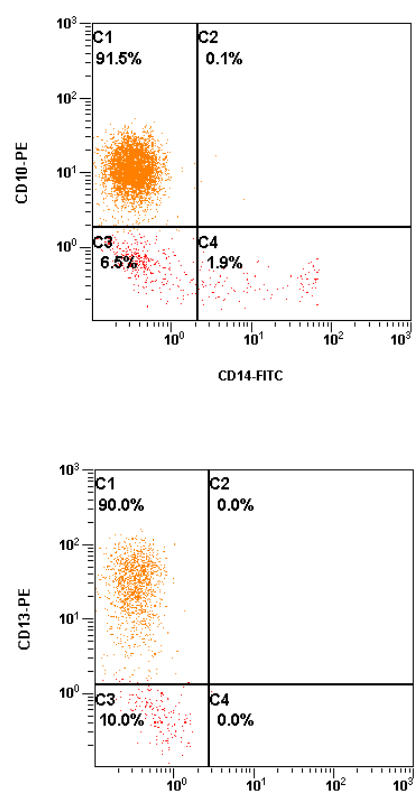

CD5-FIC
Cytogenetic analysis of the bone marrow cells showed two distinct patterns of abnormality. Among the 20 metaphase cells, 46, XY, t $(8 ; 22)$ (q24;q11.2), $\mathrm{t}(14 ; 18)(\mathrm{q} 32 ; \mathrm{q} 21)$ was detected in 6 cells, while $46 \mathrm{XY}$, $-5,-17,-20,+$ mar1, + mar2, + mar3 was detected in 3 cells (Figure 3 ). Fluorescence in situ hybridization (FISH) using dual color probes for IgH/BCL2 performed on the bone marrow cells demonstrated signal patterns indicative of an IgH/BCL2 fusion in $72 \%$ of interphase cells. FISH using IgH/MYC probes did not detect cells with this fusion pattern. In addition, FISH using single color probe for CSF1R located on chromosome $5 \mathrm{q}$ showed loss of this gene in $25 \%$ of interphase cells. FISH of IgL/MYC or IgK/MYC was not performed due to insufficient materials.

Based on the morphology, FCM, and cytogenetic analysis, he was diagnosed as DHL with bone marrow involvement complicated with AML with myelodysplasia-related changes. Since both diseases were predicted to respond poorly to anti-neoplastic therapy and he was intolerable to aggressive therapy due to his age, he was only treated supportively. Dyspnea due to pulmonary infiltration of malignant cells gradually developed, and he died of progression of DHL and AML on the $47^{\text {th }}$ hospital day.
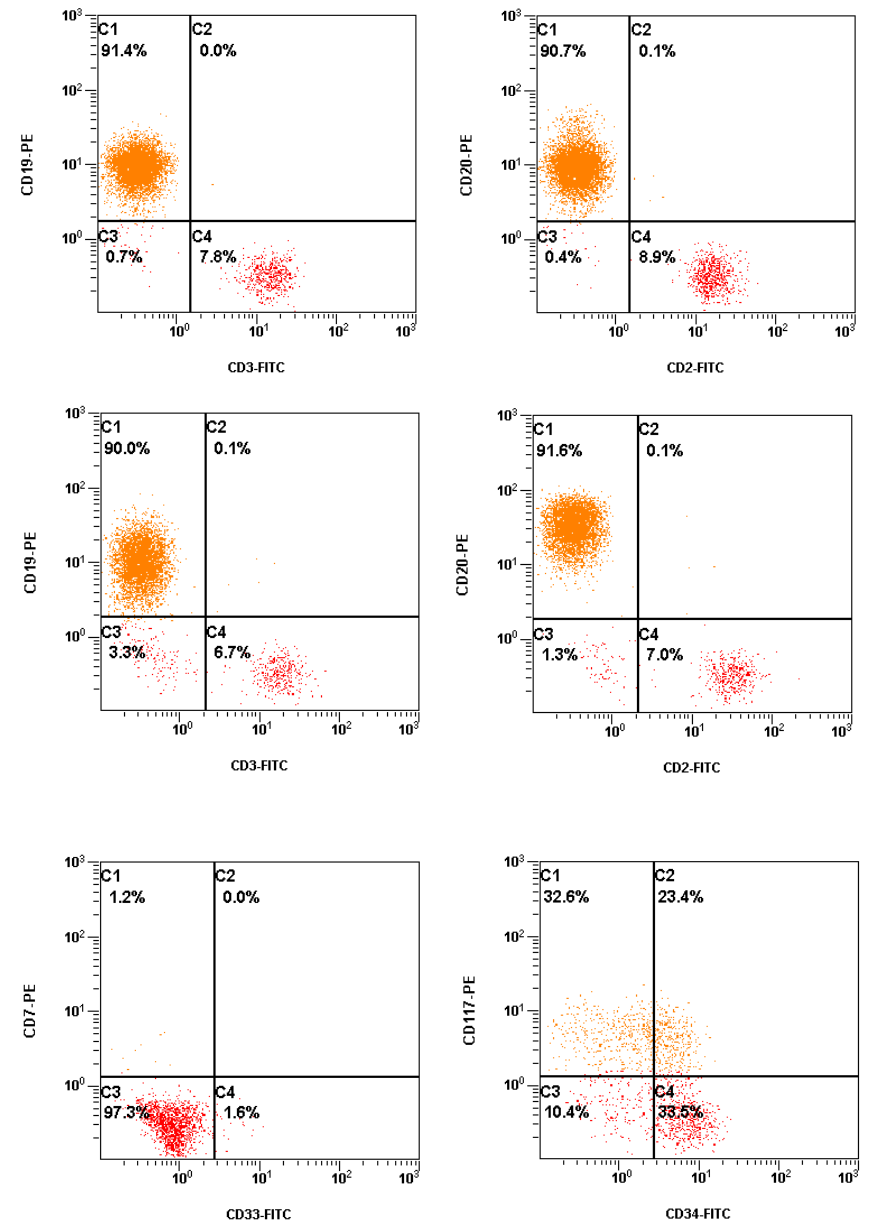

Figure 2: Flow cytometric analysis of the bone marrow cells.

$A$ and B: In the area of CD45-positive cells, two cell populations were identified. One population consisted of small lymphocytes, which were positive for CD19 and CD20, and dimly positive for CD10 (A). Another population consisted of medium-sized to large lymphocytes, which were positive for CD10, CD19, and CD20 (B). C: In the area of CD45- dimly positive cells, a distinct population of medium-sized to large cells positive for CD13, CD34, and CD117 were detected. 


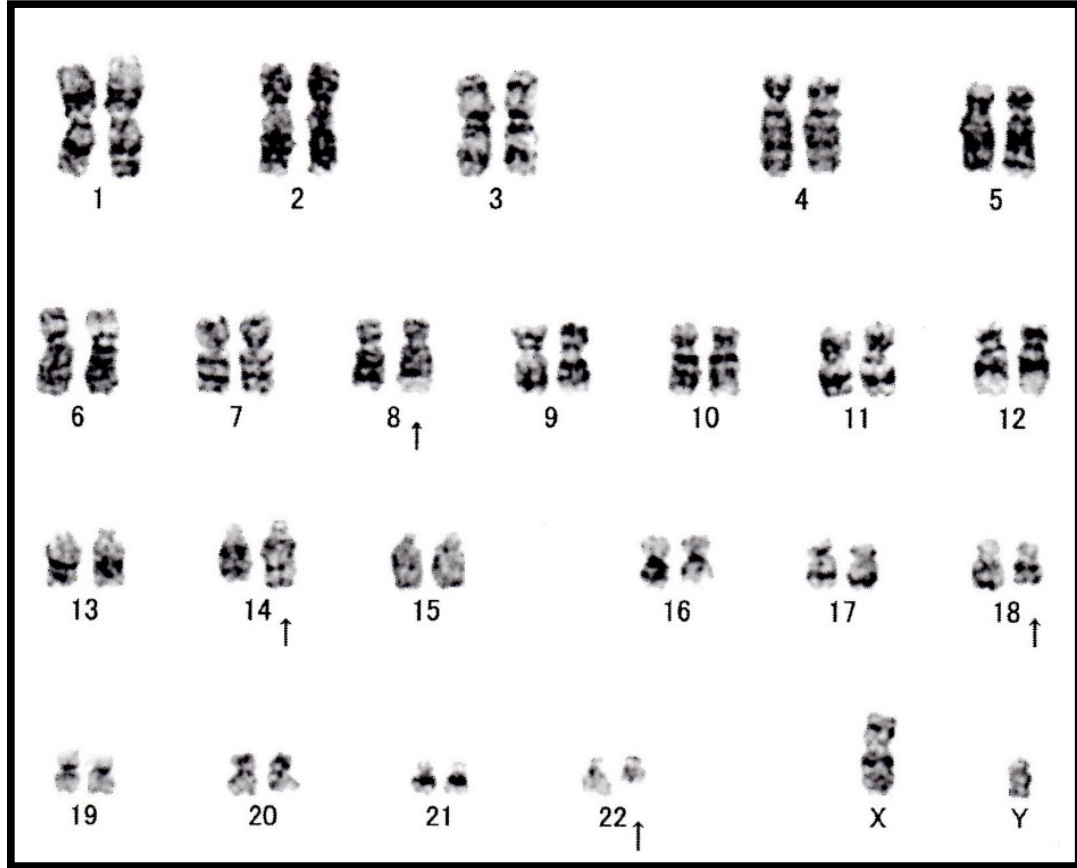

A
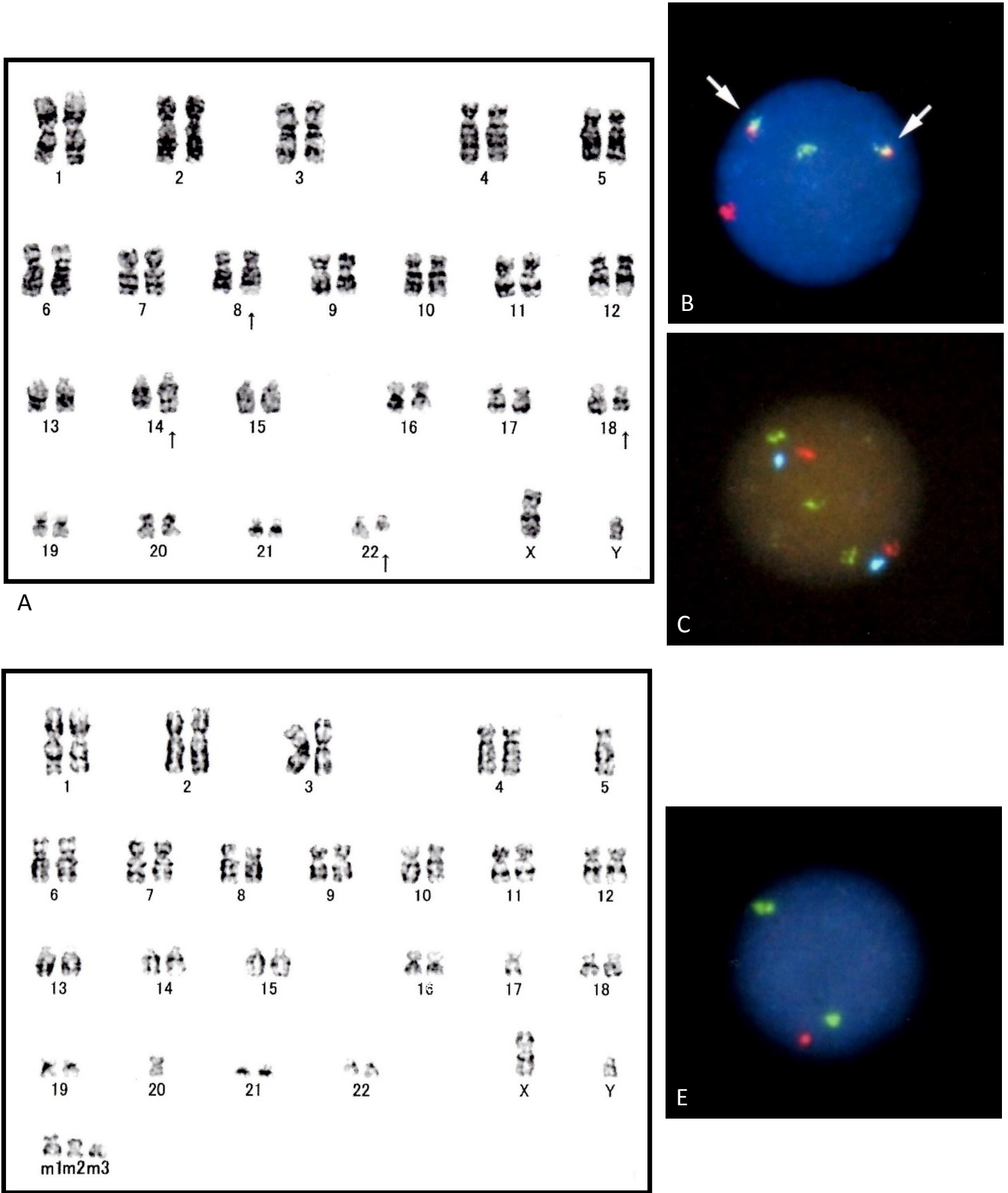

D

Figure 3: Cytogenetic analysis of the bone marrow cells

A and D: 46,XY,t(8;22)(q24;q11.2),t(14;18)(q32;q21) in 6 cells (A) and G-banding karyotype showed 46,XY,-5,-17,-20,+mar1,+mar2,+mar3 in 3 cells (D) out of 20 metaphase cells. Arrows in A indicate the chromosomes with translocation.

$\mathrm{B}, \mathrm{C}$ and $\mathrm{E}$ : FISH analyses of $\mathrm{IgH} / \mathrm{BCL} 2$ fusion (B), IgH/MYC fusion (C), and CSF1R (E). Arrows in B indicate the presence of fusion of red (IgH probe) and green (BCL2 probe) signals. No fusion of IgH/MYC was detected in C (red: MYC probe, green: IgH probe, blue: D8Z2 probe). Heterozygous loss of CSF1R (red signal) on the $5 q$ was shown in E, while D5S721 \& D5S23 (green) on the 5p of both alleles were retained. 
Citation: Sakamoto D, Satoh N, Ohnishi H, Yamasaki S, Watanabe T, et al. (2017) Simultaneous Occurrence of Double-Hit Lymphoma and Acute Myeloid Leukemia in Bone Marrow. J Blood Lymph 7: 188. doi: 10.4172/2165-7831.1000188

\section{Discussion}

The present case is, to the best of our knowledge, the first reported case with concurrence of DHL and AML. Oki et al. reported 129 cases of DHL in single institute between 2003-2013, among which 16 cases (12\%) showed leukemic presentation [5]. However, all of the cases were classified as bone marrow involvement of DHL cells, and concurrence of myeloid malignancies has not been described. In the present case, FCM analysis revealed the presence of distinct lymphoid cells (two populations) and myeloid blasts in both bone marrow and peripheral blood. Further cytogenetic analysis confirmed the presence of two distinct cell populations, one with features of DHL (concurrent $t(8 ; 22)$ $(\mathrm{q} 24 ; \mathrm{q} 11.2)$ and $\mathrm{t}(14 ; 18)(\mathrm{q} 32 ; \mathrm{q} 21))$ and another with a myelodysplasiarelated feature (loss of chromosomes 5). Consequently, FCM and cytogenetic analysis confirmed very rare concurrence of DHL and AML in this patient. Based on the distinct immunophenotypic and cytogenetic features of each cell population, we suppose that this concurrence would be incidental rather than cellular transformation from one to another. However, the possibility remains that both malignant cells derived from common leukaemic/lymphoma stem cells with unidentified genetic abnormalities. While such concurrence may be a very rare event, precise FCM and cytogenetic analysis would be useful in accurately diagnosing bone marrow involvement of DHL.

In the present case, MYC translocation was not proven by FISH analysis, but the presence of $\mathrm{t}(8 ; 22)(\mathrm{q} 24 ; \mathrm{q} 11.2)$ by cytogenetic analysis strongly suggested that the lymphoma cells harbored MYC/IgL fusion gene in addition to IgH/BCL2 fusion. Furthermore, two distinct populations of lymphoid cells were detected in the bone marrow, one of which showed morphological features of follicular lymphoma cells. Therefore, transformation from follicular lymphoma to DHL was also suspected, as has been reported in a subset of DHL cases. Actually, Kishimoto et al. reported a DHL case with two distinct lymphoma cell populations in the bone marrow having translocations of both MYC and BCL2 [9]. In this case, single BCL2 translocation was detected in the primary lymph node, suggesting the transformation from follicular lymphoma with BCL2 translocation to B-lymphoblastic leukemia with additional MYC translocation. Unfortunately, no evidence of transformation was available in our case, because cytogenetic analysis did not detect bone marrow cells with single $t(14 ; 18)(\mathrm{q} 32 ; \mathrm{q} 21)$ translocation. Cytogenetic analysis of the primary lymph node lesions might have provided more information regarding this issue. Because of wide variation in biology and morphological aspects of DHL, it is essential to fully perform cytogenetic analysis and immunophenotyping for accurate evaluation of DHL.

\section{References}

1. Fiedler W, Weh HJ, Zeller W, Fonatsch C, Hillion J, et al (1991) Translocation $(14 ; 18)$ and $(8 ; 22)$ in three patients with acute leukemia/lymphoma following centrocytic/centroblastic non-Hodgkin's lymphoma. Ann Hematol 63: 282-287.

2. Kaplan A, Samad A, Dolan MM, Cioc AM, Holman CJ, et al. (2012) Follicular lymphoma transformed to "double-hit" B lymphoblastic lymphoma presenting in the peritoneal fluid. Diagn Cytopathol 41: 986-990.

3. Tanaka H, Hashimoto S, Abe D, Sakai S, Takagi T (2011) Double-hit lymphoma at second relapse of Burkitt-like lymphoma. J Clin Exp Hematop 51: 43-47.

4. Aukema SM, Siebert R, Schuuring E, van Imhoff GW, Kluin-Nelemans HC, et al. (2011) Double-hit B-cell lymphomas. Blood 117: 2319-2331.

5. Oki Y, Noorani M, Lin P, Davis RE, Neelapu SS, et al. (2014) Double hit lymphoma: the MD Anderson Cancer Center clinical experience. $\mathrm{Br} \mathrm{J}$ Haematol 166: 891-901.

6. Tomita N (2011) BCL2 and MYC dual-hit lymphoma/leukemia. J Clin Exp Hematol 51: 7-12.

7. Yoshida M, Ichikawa A, Miyoshi H, Kiyasu J, Kimura Y, et al. (2015) Clinicopathological features of double-hit B-cell lymphomas with MYC and BCL2, BCL6 or CCND1 rearrangements. Pathol Int 65: 519-527.

8. Swerdlow SH, Campo E, Harris NL, Jaffe ES, Pileri SA, et al. (2008) WHO Classification of Tumours of Haematopoietic and Lymphoid Tissues (4th edn.), Lyon: IARC Press.

9. Kishimoto $\mathrm{W}$, Shirase T, Chihara D, Maeda T, Arimoto-Miyamoto K, et al (2012) Double-hit lymphoma with a feature of follicular lymphoma concurren with clonally related $\mathrm{B}$ lymphoblastic leukemia : A preference of transformation for the bone marrow. J Clin Exp Hematol 52: 113-119. 